\title{
An Error Analysis for Numerical Multiple Integration. III
}

\author{
By Robert E. Barnhill and Gregory M. Nielson
}

\begin{abstract}
Asymptotic results of two types are given for minimum norm cubatures, defined on a certain Hilbert space of analytic functions. Cubatures with a high degree of precision are realized as the limits of minimum norm cubatures, and an algebraic approach for the determination of efficient rules is derived. Numerical methods and examples are also included.
\end{abstract}

1. Introduction. This paper is a continuation of earlier work [2], and it concerns bounds on the cubature error for a class of uniformly bounded analytic functions of two variables. Minimum norm (MN) and optimal cubatures have been defined and discussed previously. This paper contains additional asymptotic results concerning them, as well as numerical results. The methods for doing the numerical calculations are described in some detail, both because they have changed considerably from the methods used earlier, and because we believe them to be "optimal." One of the asymptotic results given in this paper is that, in certain cases, efficient cubature rules are the limits of MN rules, and the algebraic proof of this result should be useful for determining new efficient rules.

2. Asymptotic Properties of MN Cubatures. The Hilbert space of analytic functions to be considered is $L^{2} \equiv\left\{f(z, w): f\right.$ is analytic inside $E_{\rho} \times E_{\rho}$ and $\|f\|^{2} \equiv$ $\iiint \int_{E_{n} \times E_{n}}|f(z, w)|^{2} d x d y d u d v$ exists $\}$, where $z=x+i y, \quad w=u+i v, \quad E_{\rho}$ is the ellipse with foci \pm 1 , semimajor axis $a$, semiminor axis $b=\left(a^{2}-1\right)^{1 / 2}$, and $\rho=$ $(a+b)^{2}$. With $I=[-1,1]$, we define

$$
R_{l}(f)=\iint_{i, l} f(x, u) d x d u-\sum_{k=1}^{l} A_{k} f\left(x_{k}, u_{k}\right)
$$

A MN cubature with $l$ nodes is a cubature with weights $A_{k}$ and nodes $\left(x_{k}, u_{k}\right)$ such that $\left\|R_{l}\right\|$ is a minimum. It is of interest to have an upper bound on $\left\|R_{l}^{\mathrm{MN}}\right\|$ in terms of $l$. One such upper bound appears in [2]. Let $R_{m n}^{T}$ be the remainder of the $(m \times n)$ tensor product ("cross product") Gaussian rule, with $l \geqq m n$. Of course, $\left\|R_{l}^{\mathrm{MN}}\right\| \leqq$ $\left\|R_{m n}^{G}\right\|$ and Theorem 1 contains an upper bound on $\left\|R_{m n}^{G}\right\|$.

THEOREM 1. In the space $L^{2}$

$$
\left\|R_{m n}^{G}\right\|^{2} \leqq \sum_{\substack{r=2 m \\ r \text { and s eren }}}^{x} \sum_{\substack{s=2 n \\ \text { s eren }}}^{x} \chi(r, \rho) \alpha(s, \rho)\left[\gamma_{m} \delta_{r}^{2 m} \delta_{r}^{0}+\gamma_{n} \delta_{r}^{0} \delta_{s}^{2 n}+\gamma_{m} \gamma_{n} \delta_{r}^{2 m} \delta_{s}^{2 n}\right]^{2},
$$

where

Received February 4. 1969.

AMS Suhject Classifications. Primary 4144. 6555, 6580: Secondary 6520

Key Words and Phrases. Cubature. numerical integration, approximate integration, error bounds. 


$$
\alpha(r, \rho)=4(r+1) /\left[\pi\left(\rho^{r+1}-\rho^{-r-1}\right)\right], \quad \gamma_{m}=\frac{2^{2 m+1}(m !)^{4}}{(2 m+1)[(2 m) !]^{3}}
$$

and

$$
\delta_{r}^{i}=\frac{(r+1)\left[(r+1)^{2}-1\right] \cdots\left[(r+1)^{2}-i^{2}\right]}{1 \cdot 3 \cdots(2 i+1)} .
$$

Proof.

$$
\left\|R_{m n}^{G}\right\|^{2}=\sum_{r=0}^{\infty} \sum_{s=0}^{\infty} \alpha(r, \rho) \alpha(s, \rho)\left|R_{m n}^{G}\left(U_{r}(x) U_{s}(u)\right)\right|^{2},
$$

where $\alpha(r, \rho)$ is as above and $U_{r}$ is the $r$ th Chebyshev polynomial of the second kind. Stancu [11] has shown that

$$
\begin{aligned}
R_{m n}^{G}(f)= & \frac{P_{M}}{(M+1) !} f_{M+1,0}\left(\varepsilon, \eta_{1}\right)+\frac{Q_{N}}{(N+1) !} f_{0, N+1}\left(\varepsilon_{1}, \eta\right) \\
& -\frac{P_{M} Q_{N}}{(M+1) !(N+1) !} f_{M+1, N+1}(\varepsilon, \eta),
\end{aligned}
$$

where

$$
P_{M}=(d-c) \int_{a}^{b} \prod_{i=1}^{m}\left(x-x_{i}\right)^{2} d x>0, \quad Q_{N}=(b-a) \int_{c}^{d} \prod_{j=1}^{n}\left(y-y_{j}\right)^{2} d y>0
$$

$\varepsilon$ and $\varepsilon_{1}$ are in $[a, b]$, and $\eta_{1}$ and $\eta$ in $[c, d]$. We have $a=c=-1, b=d=1$, $M=2 m-1$, and $N=2 n-1$, the latter being the respective precisions of the Gaussian quadratures. It turns out that

$$
P_{2 m-1}=\frac{2^{2 m+1}(m !)^{4}}{(2 m+1)[(2 m) !]^{2}} \equiv(2 m) ! \gamma_{m}
$$

and

$$
Q_{2 n-1}=\frac{2^{2 n+1}(n !)^{4}}{(2 n+1)[(2 n) !]^{2}} \equiv(2 n) ! \gamma_{n}
$$

Thus,

$$
\begin{aligned}
\left|R_{m n}^{G}\left(U_{r}(x) U_{s}(u)\right)\right| \leqq \gamma_{m} \mid & U_{r}^{(2 m)}(\varepsilon) U_{s}\left(\eta_{1}\right) \mid \\
& +\gamma_{n}\left|U_{r}\left(\varepsilon_{1}\right) U_{s}^{(2 n)}(\eta)\right|+. \gamma_{m} \gamma_{n}\left|U_{r}^{(2 m)}(\varepsilon) U_{s}^{(2 n)}(\eta)\right|
\end{aligned}
$$

Now

$$
\left|U_{r}^{(i)}(\varepsilon)\right| \leqq \frac{(r+1)\left[(r+1)^{2}-1\right] \cdots\left[(r+1)^{2}-i^{2}\right]}{1 \cdot 3 \cdots(2 i+1)}, \quad-1 \leqq \varepsilon \leqq 1,
$$

for $i=0,1, \cdots$. (The empty product is interpreted as the number 1.) Let the upper bound in (5) be denoted by $\delta_{r}^{i}$. Then we observe that $\delta_{r}^{0}=(r+1)$ and $\delta_{r}^{i}=0$ if $i>r$, because $U_{r}$ is a polynomial of degree $r$. The substitution of (5) into (4) and (4) into (2) yields the desired result. Q.E.D.

We note that $\left\|R_{m n}^{G}\right\|^{2}$ is $O\left(\rho^{-(m+1)} \rho^{-(n+1)}\right)=O\left(\rho^{-(m+n+2)}\right)$ for functions of two 
variables. A similar analysis for functions of $k$ variables yields $\left\|R_{m_{1} m_{2} \cdots m_{k}}\right\|^{2}=$ $O\left(\rho^{-\left(m_{1}+\cdots+m_{k}+k\right)}\right)$.

Stancu's article concerns tensor product rules, and so the error bounds of Theorem 1 are "rectangular" in form. Thus, the error formula (3) is applicuble in spaces with polynomial precision in each variable separately. The corresponding space is called $B_{\lceil 2 m, 2 n\rceil}$ by Sard [9]. Secondly, the error bound in (1) would be improved, if only the third term of (3) were present. This is exactly what is obtained by the use of Gordon's "blending functions" [5]. The blending function of interest here is the following:

$$
B(x, u)=\sum_{i=1}^{m} f\left(x_{i}, u\right) l_{i}(x)+\sum_{j=1}^{n} f\left(x, u_{j}\right) m_{j}(u)-\sum_{i=1}^{m} \sum_{j=1}^{n} f\left(x_{i}, u_{j}\right) l_{i}(x) m_{j}(u),
$$

where $l_{i}(x)=\prod_{k \neq i}\left(\left(x-x_{k}\right) /\left(x_{i}-x_{k}\right)\right)$ and $m_{j}$ dually. This function interpolates $f$ along "lines," i.e., $B\left(x_{i}, u\right)=f\left(x_{i}, u\right), i=1, \cdots, m$ for all $u$ in $I$ and $B\left(x, u_{j}\right)=f\left(x, u_{j}\right)$, $j=1, \cdots, n$ for all $x$ in $I$.

3. Asymptotic-in- $\rho$ Properties of MN Cubatures. A cubature rule is said to be efficient if it integrates exactly as many of the low order monomials as there are parameters in the rule; e.g., for a two-dimensional cubature with $n$ nodes, there are $3 n$ parameters, $n$ weights and $n$ nodes, each of which contains two parameters. Also, there are

$$
\left(\begin{array}{c}
d+2 \\
2
\end{array}\right)=\frac{(d+2)(d+1)}{2}
$$

monomials in two variables of degree $\leqq d$. For functions of one variable, Gaussian quadratures are efficient. Since the nodes of these quadratures are the zeros of the related orthogonal polynomials, various authors [6], [7], [8] have tried to use the common zeros of sets of orthogonal polynomials in more than one variable as the nodes for efficient rules. Stroud [10] has recently obtained sufficient conditions for the common zeros of orthogonal polynomials to be the nodes of a cubature with a certain precision, but the formulas so discussed need not be efficient. We remark that Stroud's article also contains a good bibliography on the subject of orthogonal polynomials and their relation to cubature theory.

For functions of one variable, it is known [2] that the $\mathrm{MN}$ weights and nodes converge to the Gaussian weights and nodes as $\rho \rightarrow \infty$. For functions of two variables, if the nodes are an interpolating set, [3, p. 27], and the number of them is of the form

$$
\left(\frac{1}{3}\right)\left(\begin{array}{c}
d+2 \\
2
\end{array}\right)
$$

for some positive integer $d$, then the weights converge to the corresponding interpolatory cubature weights [2] and, in fact, the order of convergence is $\rho^{-(d+1)}$. A similar result is known for functions of $n$ variables, $n \geqq 2$. We conjectured that the $\mathrm{MN}$ cubature nodes converge to the nodes of an efficient cubature. If so, then we would have a computationally feasible way of finding efficient cubatures. We have obtained only partial results, both theoretically and numerically. Our numerical evidence to date (see the Tables) has indicated that the above conjecture is not true in the generality stated. 
We shall state the theoretical result in a special case for clarity of the ideas; the generalization follows without difficulty. Radon [8] has discovered an efficient sevenpoint formula of precision 5 , in two variables. The cubature nodes are the common zeros of three polynomials, each of degree three. Let the region of integration be the square $S:-1 \leqq x, y \leqq 1$.

THEOREM 2. Let the cubature formula be

$$
\iint_{S} f(x, y) d x d y \simeq \sum_{k=1}^{7} A_{k} f\left(x_{k}, y_{k}\right) .
$$

Assume that (1) the MN nodes form an interpolating set (at least for all $\rho \geqq$ some $\rho^{*}$ ), and (2) the common zeros of the sample polynomials (this term is defined in the proof), corresponding to the MN nodes and to some efficient rule, are all continuous functions of the coefficients of these polynomials. Then the MN nodes converge to the nodes of an efficient rule as $\rho \rightarrow \infty$.

Remark. A key assumption is that there exists an efficient rule (whether known explicitly or not), and the Radon rule validates this assumption for this case. Note, however, that Theorem 2 does not imply that the MN cubature necessarily converges to the Radon cubature.

Proof. 1. We denote the remainder of the Radon rule by $R_{7}^{R}$. Then

$$
\left\|R_{7}^{\mathrm{MN}}\right\|^{2} \leqq\left\|R_{7}^{R}\right\|^{2}
$$

Now

$$
\left\|R_{7}^{R}\right\|^{2}=\sum_{r+s>5} \alpha(r, \rho) \alpha(s, \rho)\left|R_{7}^{R}\left(U_{r}(x) U_{s}(y)\right)\right|^{2},
$$

where $\alpha(r, \rho)=4(r+1) /\left[\pi\left(\rho^{r+1}-\rho^{-r-1}\right)\right]=O\left(\rho^{-r-1}\right)$ as $\rho \rightarrow \infty$. The sum's index is $r$, such that $r+s>5$, because $R_{7}^{R}$ has precision $5, U_{r}$ is a polynomial of degree $r$, and $U_{s}$ dually. Thus $\left\|R_{7}^{R}\right\|^{2}=O\left(\rho^{-5-2}\right)=O\left(\rho^{-7}\right)$, whereas $\left\|R_{7}^{\mathrm{MN}}\right\|^{2}=$ $O\left(\rho^{-2}\right)$. We multiply both sides of (6) by $\rho^{2}$ and observe that

$$
\begin{aligned}
R_{7}^{\mathrm{MN}}(1)+O\left(\rho^{-1}\right) R_{7}^{\mathrm{MN}}(x) & +O\left(\rho^{-1}\right) R_{7}^{\mathrm{MN}}(y)+\cdots+O\left(\rho^{-5}\right) R_{7}^{\mathrm{MN}}\left(x^{5}\right) \\
& +\cdots+O\left(\rho^{-5}\right) R_{7}^{\mathrm{MN}}\left(y^{5}\right)=O\left(\rho^{-5}\right), \text { i.e., } \lim _{\rho \rightarrow \infty} R_{7}^{\mathrm{MN}}(1)=0 .
\end{aligned}
$$

Using this fact and multiplying successively by $\rho^{3}, \rho^{4}, \cdots, \rho^{7}$, we obtain the following result:

$$
\lim _{\rho \rightarrow \infty} R_{7}^{\mathrm{MN}}\left(x^{j} y^{k}\right)=0, \quad 0 \leqq j+k \leqq 5 .
$$

2. Equation (8) can be rewritten as the following system of 21 equations (where the integrals are each over $S$ ):

$$
\begin{aligned}
A_{1}+A_{2}+\cdots+A_{n} & =\int 1+\varepsilon_{00}, \\
A_{1} x_{1}+A_{2} x_{2}+\cdots+A_{7} x_{7} & =\int x+\varepsilon_{10}, \\
A_{1} y_{1}+A_{2} y_{2}+\cdots+A_{7} y_{7} & =\int y+\varepsilon_{01}, \\
\vdots & \\
A_{1} y_{1}^{5}+A_{2} y_{2}^{5}+\cdots+A_{7} y_{7}^{5} & =\int y^{5}+\varepsilon_{05},
\end{aligned}
$$


where the $\varepsilon_{i j}=\varepsilon_{i j}(\rho) \rightarrow 0$ as $\rho \rightarrow \infty$. These $A_{i}, x_{i}$, and $y_{i}$ are the MN values. We form three so-called "sample" $\mathrm{MN}$ third degree polynomials $P_{1}, P_{2}$, and $P_{3}$ with the $\mathrm{MN}\left(x_{i}, y_{i}\right)$ as their common zeros. We note that a general third degree polynomial in two variables has ten terms, and the requirement of seven zeros means that there are three linearly independent solutions $P_{1}, P_{2}$, and $P_{3}$. We also observe that every efficient cubature satisfies Eqs. (9), with the perturbations $\varepsilon_{i j}$ equal to zero.

3. We now perform a sequence of (reversible) algebraic steps, the upshot of which is to change (9) from a nonlinear system, with perturbations tending to zero, to a rectangular linear system. The idea is to develop a two-dimensional analog of the so-called "algebraic" approach of Kopal [10] to Gaussian quadratures. Consider

$$
P_{1}(x, y)=\sum_{0 \leqq i+j \leqq 3} c_{i j} x^{i} y^{j},
$$

and assume that $P_{1}$ has some cubic term, say $x^{3}$. Then, without loss of generality, we may assume that $c_{30}=1$.

(i) Multiply the first equation of (9) by $c_{00}$, the second by $c_{10}, \cdots$, the last by $c_{03}$.

$$
\begin{aligned}
c_{00}\left[A_{1}+\cdots+A_{7}\right] & =c_{00}\left[\int 1+\varepsilon_{00}\right], \\
c_{10}\left[A_{1} x_{1}+\cdots+A_{7} x_{7}\right] & =c_{10}\left[\int x+\varepsilon_{10}\right], \\
\vdots & \\
1 \cdot\left[A_{1} x_{1}^{3}+\cdots+A_{7} x_{7}^{3}\right] & =1 \cdot\left[\int x^{3}+\varepsilon_{30}\right], \\
c_{21}\left[A_{1} x_{1}^{2} y_{1}+\cdots+A_{7} x_{7}^{2} y_{7}\right] & =c_{21}\left[\int x^{2} y+\varepsilon_{21}\right], \\
\vdots & \\
c_{03}\left[A_{1} y_{1}^{3}+\cdots+A_{7} y_{7}^{3}\right] & =c_{03}\left[\int y^{3}+\varepsilon_{03}\right] .
\end{aligned}
$$

Add these equations and factor each of the $A_{i}$ to get:

$$
\begin{aligned}
A_{1}\left[c_{00}+\right. & \left.c_{10} x_{1}+\cdots+1 \cdot x_{1}^{3}+\cdots+c_{03} y_{1}^{3}\right]+A_{2}[\cdot]_{2}+\cdots+A_{7}[\cdot]_{7} \\
& =c_{00}\left[\int 1+\varepsilon_{00}\right]+\cdots+1 \cdot\left[\int x^{3}+\varepsilon_{30}\right]+\cdots+c_{03}\left[\int y^{3}+\varepsilon_{03}\right],
\end{aligned}
$$

where $[\cdot]_{i}$ means the bracketed expression following $A_{1}$ with the substitution $x_{i}=x_{1}$. Thus, the left-hand side of this equation is zero, since the $\left(x_{i}, y_{i}\right)$ are zeros of $P_{1}$. If we regard the $c_{i j}$ as the unknowns, then (10.1) is one equation in nine unknowns.

(ii) To obtain a second equation, we multiply different equations by the same constants as before to get the following:

$$
\begin{aligned}
c_{00}\left[A_{1} x_{1}+\cdots+A_{7} x_{7}\right] & =c_{00}\left[\int x+\varepsilon_{10}\right], \\
c_{10}\left[A_{1} x_{1}^{2}+\cdots+A_{7} x_{7}^{2}\right] & =c_{10}\left[\int x^{2}+\varepsilon_{20}\right], \\
c_{01}\left[A_{1} x_{1} y_{1}+\cdots+A_{7} x_{7} y_{7}\right] & =c_{01}\left[\int x y+\varepsilon_{11}\right], \\
\vdots & \\
1 \cdot\left[A_{1} x_{1}^{4}+\cdots+A_{7} x_{7}^{4}\right] & =1 \cdot\left[\int x^{4}+\varepsilon_{40}\right], \\
\vdots & \\
c_{03}\left[A_{1} x_{1} y_{1}^{3}+\cdots+A_{7} x_{7} y_{7}^{3}\right] & =c_{03}\left[\int x y^{3}+\varepsilon_{13}\right] .
\end{aligned}
$$


Add these equations and factor each of the $A_{i} x_{i}$ to get:

$$
\begin{aligned}
A_{1} x_{1}\left[c_{00}+c_{10} x_{1}+\cdots+c_{03} y_{1}^{3}\right] & +A_{2} x_{2}[\cdot]_{2}+\cdots+A_{7}[\cdot]_{7} \\
= & c_{00}\left[\int x+\varepsilon_{10}\right]+\cdots+c_{03}\left[\int x y^{3}+\varepsilon_{13}\right] .
\end{aligned}
$$

Again, the left-hand side equals zero, and so we have a second equation.

(iii) This argument can be continued exactly, so long as the monomials involved are of total degree $\leqq 5$. If we consider (10.2) as coming from a "multiplication by $x$ " of (10.1), then the same procedure can be followed for multiplication by $1, x, y, x^{2}, x y$, and $y^{2}$, the first two yielding (10.1) and (10.2), respectively. Thus we obtain six equations in nine unknowns. Each solution of the unperturbed (i.e., all the $\varepsilon_{i j}=0$ ) (10.1), (10.2), , , (10.6) is a polynomial $R_{1}(x, y)$ with leading term $x^{3}$ and with roots (at least) at $\left\{\left(x_{i}, y_{i}\right)\right\}_{i=1}^{7}$, such that (9) holds with the $\varepsilon_{i j}=0$. Since the unperturbed equations have a solution, by the assumption of the existence of an efficient cubature, the solutions of the perturbed system are continuous in the perturbations, and thus

$$
c_{i j}^{\mathrm{MN}} \rightarrow c_{i j}^{\text {efficient }} \quad \text { as } \rho \rightarrow \infty .
$$

The zeros of $P_{1}$ and $R_{1}$ are both continuous functions of their coefficients, by hypothesis, and so the zeros of $P_{1}$ approach the zeros of $R_{1}$. (Warning: $R_{1}$ need not be one of the Radon orthogonal polynomials. See the tables.) Similar arguments applied to $P_{2}$ and $P_{3}$ yield the conclusion. Q.E.D.

Remarks on Theorem 2. The algebraic approach, developed in part 3 of the proof, does not depend on there being exactly seven nodes and, in fact, the same approach can be used to obtain a formula of precision 5 with a minimal number of points. Theorem 2 can be generalized to functions of more than two variables, to more general regions of integration, and to other degrees of precision. If the degree of precision in $n$ variables is to be $d$, then we show that

$$
\lim _{\rho \rightarrow \infty} R\left(U_{r_{1}} \cdots U_{r_{n}}\right)=0 \text { for } r_{1}+r_{2}+\cdots+r_{n} \leqq d,
$$

just as before. The number of sample polynomials depends on the number of nodes as in part 2 of the proof, and the number of equations corresponding to (9) is

$$
\left(\begin{array}{c}
d+n \\
n
\end{array}\right)
$$

for precision $d$ in $n$ dimensions.

Symmetry of the $M N$ Rules. In the numerical examples calculated, we have observed that MN rules frequently have symmetries of various types. It is not always possible to have full symmetry, i.e., symmetry around $x=0, y=0$, and $y= \pm x$, because, e.g., an efficient rule with precision 11 in two variables would require 26 points, and 26 is not divisible by four. We conjecture that the $\rho \rightarrow \infty$ limit of a MN cubature is exact for at least an efficient number of monomials. However, these are not necessarily the lowest order monomials, e.g., the 5-point rule given in Table 6 which integrates all monomials of degree $\leqq 3$ and, by symmetry, all odd monomials, but does not integrate $x^{2} y^{2}$. (To be efficient, a 5-point rule in two variables should have precision 4. However, such a rule does not exist [13].) Secondly, we conjecture that a $\rho \rightarrow \infty$ limit of MN cubatures integrates at least as many of the lowest order 
monomials as there are degrees of freedom in the cubature. These degrees of freedom are the original number of variables minus the number of symmetries.

4. Connection Between MN Cubatures and the Reproducing Kernel for $L^{2}$. The reproducing kernel function for the space $L^{2}$ is

$$
K(z, w, s, t) \equiv \sum_{r, u=0}^{\infty} p_{r}(z) p_{u}(w) \overline{p_{r}(s) p_{u}(t)}
$$

where

$$
p_{r}(z)=2\left[(r+1) / \pi\left(\rho^{r+1}-\rho^{-r-1}\right)\right]^{1 / 2} U_{r}(z) .
$$

If $R_{n}$ denotes the remainder of an $n$-point cubature, then a MN cubature minimizes $\left\|R_{n}\right\|^{2}$ and hence is a solution of the equation

$$
\nabla\left\|R_{n}\right\|^{2}=0,
$$

where the gradient is taken with respect to each weight and each coordinate of each node. After a short calculation, Eq. (11) can be rewritten as follows:

$$
\begin{aligned}
\nabla\left\|R_{n}\right\|^{2} & =\left[\begin{array}{l}
\partial\left(\left\|R_{n}\right\|^{2}\right) / \partial A_{j} \\
\partial\left(\left\|R_{n}\right\|^{2}\right) / \partial x_{j} \\
\partial\left(\left\|R_{n}\right\|^{2}\right) / \partial y_{j}
\end{array}\right] \\
& =\left[\begin{array}{l}
2 R_{(z, w)} K\left(z, w ; x_{j}, y_{j}\right) \\
2 A_{j} R_{(z, w)} \partial K\left(z, w ; x_{j}, y_{j}\right) / \partial x_{j} \\
2 A_{j} R_{(z, w)} \partial K\left(z, w ; x_{j}, y_{j}\right) / \partial y_{j}
\end{array}\right] \\
& =\left[\begin{array}{l}
2 R_{n}\left(\eta_{j}\right) \\
2 A_{j} R_{n}\left(\eta_{j}^{x}\right) \\
2 A_{j} R_{n}\left(\eta_{j}^{y}\right)
\end{array}\right]=0 ;
\end{aligned}
$$

where $R_{(z, w)}$ means that the functional $R$ applies to functions of $z$ and $w, \eta_{j}=\eta_{j}(z, w)$ is the representer of the point functional $L_{j}(f)=f\left(x_{j}, y_{j}\right)$, so that

$$
\eta_{j}(z, w)=K\left(z, w ; x_{j}, y_{j}\right)
$$

and

$$
\eta_{j}^{x}=\eta_{j}^{x}(z, w)=\partial K\left(z, w ; x_{j}, y_{j}\right) / \partial x_{j}
$$

is the representer of the functional

$$
L_{j}^{x}(f)=\partial f\left(x_{j}, y_{j}\right) / \partial x_{j},
$$

and $\eta_{j}^{y}$ is dual. Thus, Eq. (11) is equivalent to the requirement that a MN cubature be exact for the representers $\eta_{j}$ of the point functionals and the representers $\eta_{j}^{x}$ and $\eta_{j}^{y}$ of the partial derivatives of the point functionals. If the nodes are fixed and the minimization is only with respect to the weights $A_{j}$, then the requirement $R_{n}\left(\eta_{j}\right)=0$, 
$j=1, \cdots, n$ can be seen as follows: Let $L$ be the integration functional with representer $\eta$. Then

$$
\min _{A_{k}}\left\|R_{n}\right\|=\min _{A_{k}}\left\|L-\Sigma A_{k} L_{k}\right\|=\min _{A_{k}}\left\|\eta-\Sigma A_{k} \eta_{k}\right\|
$$

by the duality properties of Hilbert space. Then

$$
\left(L-\Sigma A_{k} L_{k}\right)\left(\eta_{j}\right)=\left(\eta-\Sigma A_{k} \eta_{k}, \eta_{j}\right)=0,
$$

because $\eta$ minus its best $L^{2}$-approximation by linear combinations of the $\eta_{k}$ is orthogonal to all of the $\eta_{j}$ and hence $R_{n}\left(\eta_{j}\right)=0, j=1, \cdots, n$. (Cf. Corollary 8.6.2 in Davis [3].)

\section{Numerical Results.}

(a) Method of calculation. The gradient $\nabla\left\|R_{n}\right\|^{2}$ involves infinite sums which must be truncated in the numerical calculations. Instead of solving (12) to find stationary values of $\nabla\left\|R_{n}\right\|^{2}$, for each sum we consider the infinite system of equations $R_{n}\left(\phi_{i}\right)=0$, $i=1,2, \cdots$. (The definition of $\phi_{i}$ depends on which sum is involved, of course.) This system is truncated to $R_{n}\left(\phi_{i}\right)=0, i=1, \cdots, L$ and then solved in the $l^{2}$-sense by a generalized Newton's method. A typical $L$ used was 200 . Let $z^{(k)}$ be the 3-tuple of $n$-dimensional vectors $\left(\bar{A}^{(k)}, \bar{x}^{(k)}, \bar{y}^{(k)}\right)$, and let

$$
F\left(z^{(k)}\right)=\left[R_{n}\left(\phi_{1} ; z^{(k)}\right), \cdots, R_{n}\left(\phi_{L} ; z^{(k)}\right)\right]^{T},
$$

where $R_{n}\left(\phi_{1} ; z^{(k)}\right)$ was called $R_{n}\left(\phi_{1}\right)$ above. The Newton's method used is the following:

$$
z^{(k+1)}=z^{(k)}-\left[F^{\prime}\left(z^{(k)}\right)\right]^{+} F\left(z^{(k)}\right),
$$

where $F^{\prime}\left(z^{(k)}\right)$ is the Fréchet derivative (i.e., the Jacobian) of $F$ at $z^{(k)}$ and $\left[F^{\prime}\left(z^{(k)}\right)\right]^{+}$ is its generalized inverse. Since $F^{\prime}(z)$ has full column rank $n$, the generalized inverse has the simpler form $[\cdot]^{+}=\left\{[\cdot]^{*}[\cdot]\right\}^{-1}[\cdot]^{*}$, where ${ }^{*}$ denotes the conjugate transpose.

This method of calculation has more global convergence properties [16] than the standard Newton's method which has been used in the past. This lessens the instabilities inherent in solving least squares problems. (See Gautschi [4] for a discussion of these instabilities.) In some cases the iterates changed too rapidly. When this occurred, relaxation factors were included in (13):

$$
z^{(k+1)}=z^{(k)}-\gamma_{k}\left[F^{\prime}\left(z^{(k)}\right)\right]^{+} F\left(z^{(k)}\right) .
$$

Usually $\gamma_{k} \equiv \gamma$ and $\gamma$ as small as 0.01 were used.

We add one disconcerting note: stationary values of $\nabla\left\|R_{n}\right\|^{2}$ need not correspond to minima of $\left\|R_{n}\right\|^{2}$. The 5-4 example given later illustrates this possibility.

The choice of initial approximations is difficult and was done in an ad hoc fashion for each case, usually with some symmetries assumed. The nodes were assumed to be in the region of integration. In all cases, th 2 region is $[-1,1] \times[-1,1]$, unless otherwise stated

(b) Representative Results.

Tables 1 and 2. The first example is a 7-point formula in the plane. An efficient rule has precision 5 . The column labeled $\left\|R_{7}^{\infty}\right\|^{2}$ is the square of the norm of the limit functional (when known). 
TABLE 1

$$
\text { Weight }
$$

$$
\begin{array}{lc}
A & \pm(s, r), \pm(r, s) \\
B & \pm(-t, t) \\
C & (0,0)
\end{array}
$$

\begin{tabular}{|c|c|c|c|c|c|c|c|c|}
\hline$a$ & $A$ & $r$ & $S$ & $B$ & $t$ & $C$ & $\left\|R_{7}\right\|^{2}$ & $\left\|R_{7}^{\infty}\right\|^{2}$ \\
\hline 1.5 & .427743 & .878312 & $.368882 \quad .5$ & .594762 & .682098 & 1.09801 & $.3432(-3)$ & $.3579(-3)$ \\
\hline 2.0 & .419542 & .887496 & .373003 .5 & .595426 & .682976 & 1.13096 & $.1210(-5)$ & $.1222(-5)$ \\
\hline 2.5 & .417729 & .889478 & 373799.5 & .595330 & .683081 & 1.13838 & $.2234(-7)$ & $.2242(-7)$ \\
\hline 3.0 & .417158 & .890115 & 374048.5 & .595283 & .683109 & 1.14080 & $.9732(-9)$ & $.9749(-0)$ \\
\hline 5.0 & .416726 & .890589 & 374232.5 & .595244 & .683128 & 1.14261 & $.2033(-12)$ & $.2033(-12)$ \\
\hline 6.0 & .416695 & .890623 & 374245.5 & .595241 & .683129 & 1.14272 & $.1047(-13)$ & $.1047(-13)$ \\
\hline 8.0 & .416675 & .890645 & .574253 .5 & .595239 & .683130 & 1.14282 & $.9978(-16)$ & $.9978(-16)$ \\
\hline \multirow[t]{4}{*}{$\infty$} & .416667 & .890654 & 374257.5 & .595238 & .683130 & 1.14286 & & \\
\hline & or & or & or & or & or & or & & \\
\hline & 5 & $7+\sqrt{ } 24$ & $7-\sqrt{ } 24$ & 425 & 1 & 8 & & \\
\hline & $\overline{12}$ & 15 & 15 & $\overline{42}$ & $\sqrt{15}$ & $\overline{7}$ & & \\
\hline
\end{tabular}

TABLE 2

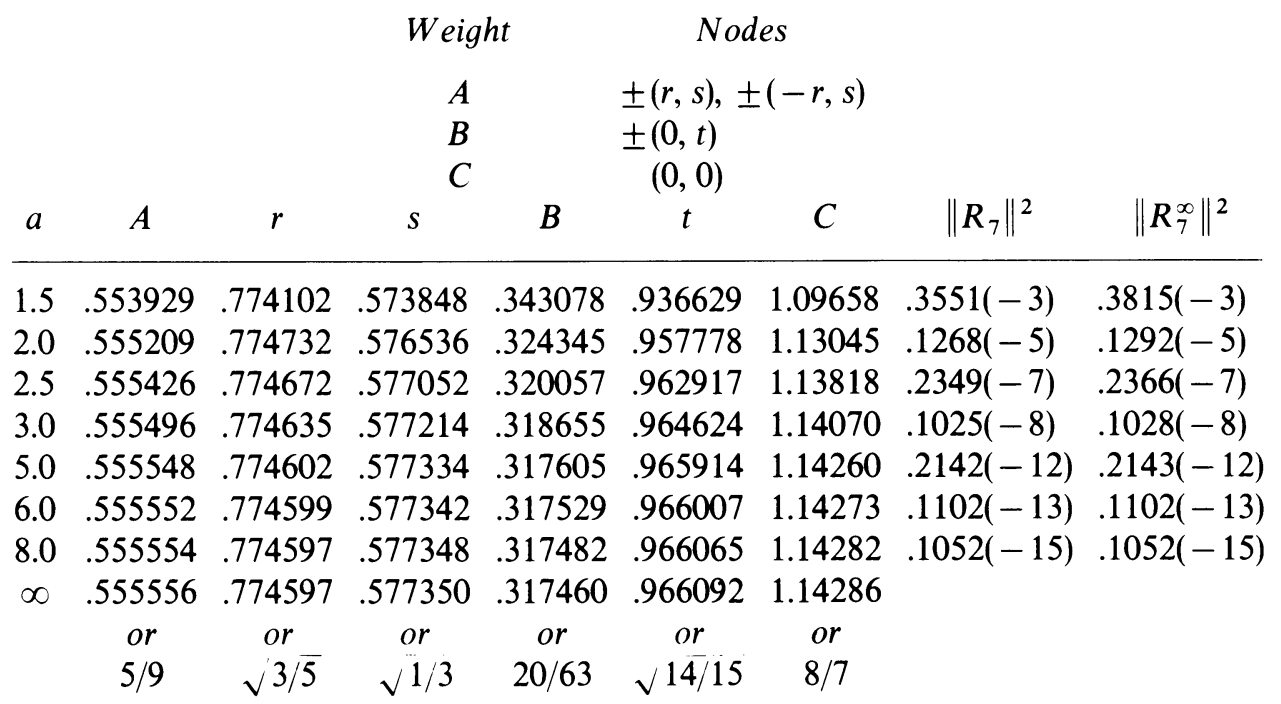

Table 3. The next example is a 12-point formula in the plane. An efficient rule has precision 7. The MN numerical results are given in Table 3. The limiting cubature, as $a \rightarrow \infty$ is an efficient rule due to Tyler [14]. There is a second such efficient rule, due to Mysovskih [12], but its nodes are not in $[-1,1] \times[-1,1]$. 
TABLE 3

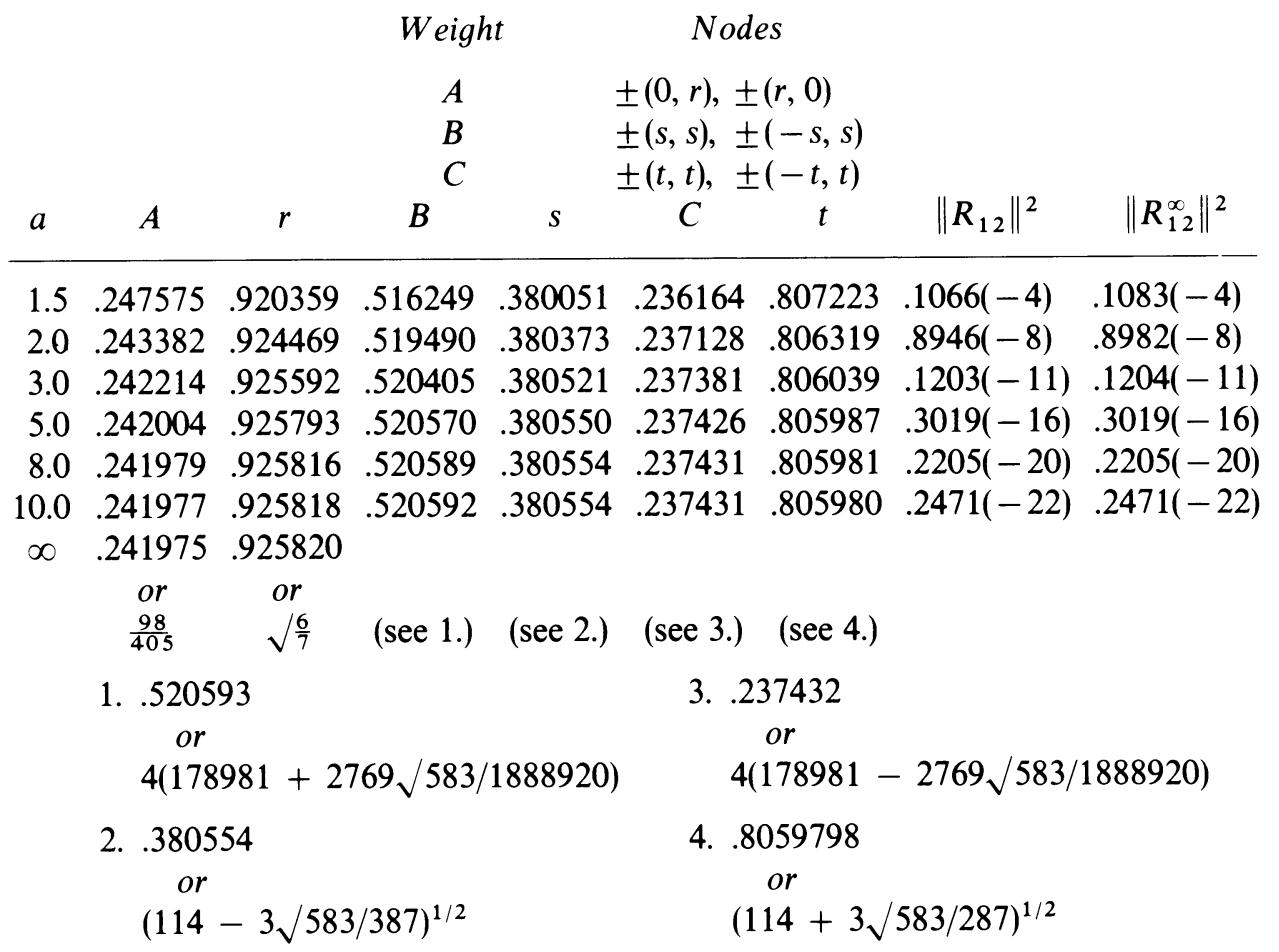

Tables 4, 5, and 6. The third example concerns 5-point formulas in the plane. An efficient rule would have precision 4 , but no efficient rules exist for this case. Three different stationary values of $\nabla\left\|R_{5}\right\|^{2}$ were found.

\section{TABle 4}

\begin{tabular}{|c|c|c|c|c|c|c|}
\hline \multirow[b]{2}{*}{$a$} & \multirow[b]{2}{*}{$A$} & \multicolumn{2}{|c|}{$\begin{array}{c}\text { Weight } \\
\text { A } \\
B\end{array}$} & $\begin{array}{l}\text { Nodes } \\
r), \pm(- \\
q)\end{array}$ & & \multirow[b]{2}{*}{$\left\|R_{5}^{\infty}\right\|^{2}$} \\
\hline & & $r$ & $B$ & $q$ & $\left\|R_{5}\right\|^{2}$ & \\
\hline 2.0 & .702832 & .687126 & 1.18795 & $10^{-15}$ & $.3483(-4)$ & $.3499(-4)$ \\
\hline 3.0 & .703570 & .688060 & 1.18569 & $10^{-16}$ & $.1623(-6)$ & $.1625(-6)$ \\
\hline 6.0 & .703696 & .688236 & 1.18522 & $10^{-15}$ & $.3032(-10)$ & $.3033(-10)$ \\
\hline 7.0 & .703699 & .688241 & 1.18520 & $10^{-15}$ & $.4661(-11)$ & $.4663(-11)$ \\
\hline 8.0 & .703701 & .688244 & 1.18519 & $10^{-14}$ & $.9256(-12)$ & $.9256(-12)$ \\
\hline$\infty$ & .703704 & .688247 & 1.18519 & 0.0 & & \\
\hline & $\begin{array}{c}\text { or } \\
19 / 27\end{array}$ & $\begin{array}{c}\text { or } \\
3 / \sqrt{ } 19\end{array}$ & $\begin{array}{c}\text { or } \\
32 / 27\end{array}$ & & & \\
\hline
\end{tabular}


TABLE 5

\begin{tabular}{|c|c|c|c|c|c|}
\hline$a$ & $A$ & $\begin{array}{l}\text { Weight } \\
\qquad \begin{array}{l}A \\
B \\
C \\
\quad S\end{array}\end{array}$ & $\begin{array}{c}\text { Nodes } \\
(s, r),(-r,-s) \\
(-t, t) \\
(v,-u),(u,-v) \\
r\end{array}$ & $B$ & $t$ \\
\hline 1.5 & .631441 & .485659 & .781122 & 1.080644 & .515539 \\
\hline 2.0 & .578063 & .497280 & .812022 & 1.146004 & .505742 \\
\hline 3.0 & .539836 & .506069 & .836316 & 1.187194 & .498267 \\
\hline 6.0 & .517174 & .511755 & .852244 & 1.211646 & .493494 \\
\hline 8.0 & .513875 & .512615 & .854671 & 1.215239 & .492773 \\
\hline 15.0 & .510841 & .513415 & .856929 & 1.218558 & .492103 \\
\hline 25.0 & .510068 & .513620 & .857509 & 1.219403 & .491932 \\
\hline 40.0 & .509804 & .513690 & .857707 & 1.219693 & .491873 \\
\hline 1000.0 & .509634 & .513735 & .857835 & 1.219879 & .491836 \\
\hline$a$ & $C$ & $u$ & $v$ & $\left\|R_{5}\right\|^{2}$ & \\
\hline 1.5 & .820605 & .139892 & .767683 & $.2895(-2)$ & \\
\hline 2.0 & .848520 & .129679 & .767892 & $.3617(-4)$ & \\
\hline 3.0 & .866556 & .121341 & .767029 & $.1579(-6)$ & \\
\hline 6.0 & .877003 & .116010 & .766578 & $.2813(-10)$ & \\
\hline 8.0 & .878504 & .115212 & .766527 & $.6821(-12)$ & \\
\hline 15.0 & .879881 & .114473 & .766485 & $.6231(-12)$ & \\
\hline 25.0 & .880230 & .114284 & .766475 & $.9333(-18)$ & \\
\hline 40.0 & .880349 & .114220 & .766471 & $.3304(-20)$ & \\
\hline 1000.0 & .880426 & .114178 & .766469 & $.5531(-37)$ & \\
\hline
\end{tabular}

TABLE 6

Weight

$\begin{array}{cc}A & \pm(0, r), \pm(r, 0) \\ B & (0,0)\end{array}$

\begin{tabular}{cccccc}
$a$ & $A$ & $r$ & $B$ & $\left\|R_{5}\right\|^{2}$ & $\left\|R_{5}^{\infty}\right\|^{2}$ \\
\hline 1.5 & 1.024699 & .773464 & -.125221 & $.6599(-2)$ & $.7462(-2)$ \\
2.0 & 1.089254 & .774336 & -.358678 & $.9915(-4)$ & $.1022(-3)$ \\
3.0 & 1.107392 & .774553 & -.429617 & $.4785(-6)$ & $.4810(-6)$ \\
6.0 & 1.110898 & .774594 & -.443591 & $.9000(-10)$ & $.9003(-10)$ \\
8.0 & 1.111044 & .774595 & -.444178 & $.2788(-11)$ & $.2788(-11)$ \\
$\infty$ & 1.111111 & .774597 & -.444444 & & \\
& or & or & or & & \\
& $10 / 9$ & $\sqrt{ } 3 / 5$ & $-4 / 9$ & &
\end{tabular}


The configuration in Table 4 is similar to one reported by Valentin [15]. It is interesting to note that the limiting cubature in Table 4 has more polynomial precision than the corresponding one in Table 5, but that the norm in the second case is smaller than in the first. The limiting cubature of Table 4 has precision 3 and enough symmetry to be exact for all monomials $x^{\alpha} y^{\beta}$ where $\alpha$ or $\beta$ is odd. The "limiting" cubature of Table 5 numerically has precision 3 , but does not have sufficient symmetry to obtain any further algebraic precision. The limiting cubature of Table 6 has sufficient symmetry to be exact for all monomial $x^{\alpha} y^{\beta}$ where $\alpha$ or $\beta$ is odd. It also is exact for the monomials $x^{2}, y^{2}, x^{4}, y^{4}$. Thus, it lacks only exactness for the monomial $x^{2} y^{2}$ of having precision 4 . Of the three rules, the one in Table 6 has the most polynomial precision, but at the expense of a negative weight.

Tables 7 and 8. We have computed two examples for the three-dimensional cube $[-1,1] \times[-1,1] \times[-1,1]$. A 5 -point precision 3 formula would be efficient, but such a formula does not exist [12]. Convergence of the Newton iterates was not obtained for this case. $13-5$ and $14-5$ formulas are known, the latter being efficient and the former hyper-efficient. The cubature corresponding to $a=\infty$ of Table 7 is a hyper-efficient formula of degree 5. It is due to Stroud [12]. The cubature corresponding to $a=\infty$ of Table 8 is an efficient formula of degree 5. It is due to Hammer and Stroud [6].

TABLE 7

\begin{tabular}{|c|c|c|c|c|c|}
\hline \multirow[b]{3}{*}{$a$} & \multicolumn{2}{|c|}{ Weight } & \multicolumn{2}{|l|}{ Nodes } & \multirow[b]{3}{*}{$C$} \\
\hline & & \multicolumn{3}{|c|}{$\begin{aligned} & (0,0,0) \\
\pm & (r, s, s), \pm(s, r, s), \pm(s, s, r) \\
\pm & (u, u, v), \pm(u, v, u), \pm(v, u, u)\end{aligned}$} & \\
\hline & $A$ & $B$ & $r$ & $s$ & \\
\hline 1.5 & 1.54223 & .563452 & .859686 & -.493456 & .511637 \\
\hline 5.0 & 1.68338 & .545106 & .880190 & -.495840 & .507664 \\
\hline 8.0 & 1.68409 & .545005 & .880287 & -.495847 & .507647 \\
\hline$\infty$ & 1.68421 & .544987 & .880304 & -.495848 & .507644 \\
\hline$a$ & $u$ & $v$ & $\left\|R_{13}\right\|^{2}$ & $\left\|R_{13}^{\infty}\right\|^{2}$ & \\
\hline 1.5 & .787474 & .030395 & $.5314(-3)$ & $.5668(-3)$ & \\
\hline 5.0 & .795586 & .025326 & $.2212(-13)$ & $.2215(-13)$ & \\
\hline 8.0 & .795616 & .025298 & $.4189(-17)$ & $.5404(-17)$ & \\
\hline$\infty$ & .795621 & .025293 & & & \\
\hline
\end{tabular}

(c) Examples. We have used the MN rules to integrate some specific functions and the numerical results are given below. Let $f(x, y) \equiv 1 /(4+x+y)$, so $\int_{-1}^{1} \int_{-1}^{1} f=$ 1.046496 , and let $g(x, y) \equiv \sin (x y)$ so that $\int_{-1}^{1} \int_{-1}^{1} g=0$. 
TABLE 8

\begin{tabular}{ccccccc} 
& & Weight & \multicolumn{4}{c}{ Nodes } \\
& & $A$ & \multicolumn{2}{c}{$( \pm r, 0,0),(0, \pm r, 0),(0,0, \pm r)$} & \\
& & $B$ & \multicolumn{2}{c}{$( \pm s, \pm s, \pm s)$} & $\left\|R_{14}\right\|^{2}$ & $\left\|R_{14}^{\infty}\right\|^{2}$ \\
\hline$a$ & $A$ & $r$ & $B$ & $s$ & & \\
\hline 1.5 & .852894 & .792192 & .359569 & .743122 & $.3900(-3)$ & $.4131(-3)$ \\
5.0 & .886234 & .795807 & .335324 & .758694 & $.1579(-13)$ & $.1580(-13)$ \\
$\infty$ & .886427 & .795822 & .335180 & .758787 & & \\
& or & or & or & or & & \\
& $\frac{320}{361}$ & $\sqrt{ } \frac{19}{30}$ & $\frac{121}{361}$ & $\sqrt{ } \frac{19}{33}$ & &
\end{tabular}

TABLE 9

\begin{tabular}{crcc}
$a$ & \multicolumn{1}{c}{$R_{7}(f)$} & $R_{12}(f)$ & \multicolumn{1}{c}{$R_{7}(g)$} \\
\hline 1.5 & -.002141 & .000019 & -0.011012 \\
2.0 & .000246 & .000003 & -0.009892 \\
3.0 & .000206 & .000003 & -0.009738 \\
5.0 & .000200 & .000003 & -0.009718 \\
$\propto$ & .000200 & .000003 & -0.009715
\end{tabular}

$R_{12}(g)=0.000000$ for the above $a$.

For the function $g(x, y)=\sin (x y)$, the $\mathrm{MN}$ error bound for the $7-5$ case is the following:

$$
\begin{gathered}
a=1.5, \\
\left\|R_{7}^{\mathrm{MN}}\right\|=1.85 \times 10^{-2} \max _{(z, w) \in E_{\nu} \times E_{\nu}}|\sin (z w)| \leqq\left|\sin \left(a^{2} i\right)\right|=\frac{1}{2}\left(e^{a^{2}}-e^{-a^{2}}\right) \equiv M .
\end{gathered}
$$

Then $\|g\| \leqq M \pi a b=24.97$ and

$$
\left|R_{7}^{\mathrm{MN}}(g)\right| \leqq\left\|R_{7}^{\mathrm{MN}}\right\| \cdot M \pi a b=46.2 \times 10^{-2} .
$$

The actual error is, from Table $9,1.10 \times 10^{-2}$, so that the error bound is conservative by only one order of magnitude. It is also interesting to compare this bound with the corresponding bound for the $7-5$ Radon cubature. If $R_{7}^{R}$ is the $7-5$ Radon rule's remainder, then $\left\|R_{7}^{R}\right\|=1.95 \times 10^{-2}$. Thus the bound $\left\|R_{7}^{R}\right\| \cdot M \pi a b$ is $1.95 / 1.85=1.054$ times what it is in the $\mathrm{MN}$ case, i.e., $48.7 \times 10^{-2}$.

Finally, the same function's $12-7 \mathrm{MN}$ bound, for $a=3$, is $\left\|R_{12}^{\mathrm{MN}}\right\|(M \pi a b)=$ $11.9 \times 10^{-2}$.

(d) Remarks on the Use of the Cubatures. $\mathrm{MN}$ cubatures have essentially the same advantages and disadvantages as efficient rules, as would be expected from Theorem 2. There are two main types of cubature rules in use currently: monomial rules and cross-product rules. Monomial rules integrate all polynomials of a certain total degree $M-1$ or less, while cross-product rules integrate all polynomials of a certain 
degree $m-1$ or less in $x$ and of a certain degree $n-1$ or less in $y$. The spaces defined by Sard [9] as $B_{m, n}$ and $B_{|m, n|}$, respectively, are the appropriate function spaces for these two possibilities. $\|R\|^{2}$, for these two cases, is $O\left(1 / \rho^{M+2}\right)$ and $O\left(1 / \rho{ }^{+2}\right)$, where $N$ is the minimum of $m$ and $n$. With respect to these 0 terms, monomial rules yield the same order of convergence as cross-product rules which involve twice as many parameters. (The corresponding Sard spaces are $B_{m, n}$ and $B_{|m+n, m+n|}$ )

6. Conclusion. Perhaps the most important feature of the $M N$ cubatures is their utility in finding efficient cubatures. Two ways of accomplishing this have been given in this paper: consider the limit as $\rho \rightarrow \infty$ of the MN cubatures or use the algebraic approach developed in the proof of Theorem 2. Different regions of integration $R$, as well as weight functions, can be used, the only restriction being that it must be possible to integrate $U_{r}(x) U_{s}(u)$ over $R$.

Acknowledgments. The first author's research was supported by the National Science Foundation by Grant GP 9021 to The University of Utah. The second author held a National Science Foundation Traineeship during his work on this research. The authors are indebted to Professor A. H. Stroud for helpful comments concerning known cubatures.

The University of Utah

Salt Lake City, Utah 84112

1. J. Albrecht \& L. Collatz, "Zur numerischen Auswertung mehrdimensionaler Integrale," $Z$. Angew. Math. Mech., v. 38, 1958, pp. 1-15. MR 20 \#432.

2. R. E. BARNHILL, "An error analysis for numerical multiple integration. I, II.," Math. Comp., v. 22. 1968. pp. 98-109: 286-292. MR 37 \#2438; MR 37 \#6027.

3. P. J. DAvis, Interpolation and Approximation, Blaisdell, Waltham, Mass., 1963. MR 28 \# 393.

4. W. Gautschl, "Construction of Gauss-Christoffel quadrature formulas," Math. Comp., v. 22, 1968, pp. 251-270. MR 37 \#3755.

5. W. J. GORDON, Blending-Function Methods of Bivariate and Multivariate Interpolation and Approximation. General Motors Research Report GMR-834, Warren, Michigan, 1968.

6. P. C. Hammer, Numerical Evaluation of Multiple Integrals, Proc. Sympos. Numerical Approximation (Madison. Wis., 1958), Univ. of Wisconsin Press, Madison, Wis., 1959, pp. 99-115. MR 20 \#6788.

7. P. M. HIRSCH, "Evaluation of orthogonal polynomials and relationship to evaluating multiple integrals," Math. Comp., v. 22, 1968, pp. 280-285. MR 37 \#2441.

8. J. RaDON, “Zur mechanischen Kubatur," Monatsh. Math., v. 52, 1948, pp. 286-300. MR 11, 405.

9. A. Sard, Linear Approximation, Math Surveys, no. 9, Amer. Math. Soc., Providence, R. I., 1963 MR 28 \# 1429.

10. A. H. Stroud, "Integration formulas and orthogonal polynomials," SIAM J. Numer. Anal., v. 4. 1967, pp. 381-389. MR 37 \#3764.

11. D. D. STANCU, "The remainder of certain linear approximation formulas in two variables," SIAM J. Numer. Anal. Ser. B, v. 1, 1964, pp. 137-163. MR 31 \# 1503.

12. A. H. Stroud, Approximate Calculation of Multiple Integrals. (Manuscript.)

13. A. H. STroud, "Quadrature methods for functions of more than one variable," Ann. New York Acad. Sci., v. 86, 1960, pp. 776-787. MR 22 \#10179.

14. G. W. TYler, "Numerical integration of functions of several variables," Canad. J. Math., v. 5, 1953, pp. 393-412. MR 15, 67.

15. R. A. VAlentin, Applications of Functional Analysis to Optimal Approximation for Analytic Functions, Ph.D. Thesis, Division of Applied Math., Brown Univ., Providence, R. I., 1965.

16. G. M. Nielson, Nonlinear Approximations in the $l_{2}$ Norm, M.S. Thesis, Department of Mathematics, Univ. of Utah, Salt Lake City, Utah, 1968. 